\title{
Synthesis and Properties of Silicon-containing Polymers Having Cyclic Silphenylenesiloxane Moieties
}

\author{
Keita Noguchi $^{1,2}$, Eiichi Akiyama ${ }^{1}$, Hitoshi Ito $^{3}$, Yu Nagase ${ }^{2}$ \\ ${ }^{1}$ Sagami Chemical Research Center, 2743-1 Hayakawa, Ayase, Kanagawa 252-1193, Japan \\ Fax: +81-467-77-4113,*e-mail: akiyama@sagami.or.jp \\ ${ }^{2}$ Tokai University, 1117 Kitakaname, Hiratsuka, Kanagawa 259-1292, Japan \\ ${ }^{3}$ Ebara Research Co. Ltd., 4-2-1 Hon-fujisawa, Fujisawa, Kanagawa 251-8502, Japan
}

\begin{abstract}
The spiro-type polymer having alternate structure of cyclic silphenylenesiloxane and cyclic carbosilane moieties was synthesized by the hydrosilylation polymerization of cyclic silphenylenesiloxane derivative having two allylhydrosilyl groups. The thermal properties of the obtained polymers were investigated by using the thermogravimetric analysis and the differential scanning calorimetry. The obtained spiro-type polymer, P3, exhibited the relatively high glass transition temperature of $77^{\circ} \mathrm{C}$ and the weight residue at $1000^{\circ} \mathrm{C}$ of $73 \%$. In addition, P3 annealed at $200^{\circ} \mathrm{C}$ was an excellent heat-resistant material insoluble to common solvents. The dielectric constants, $\varepsilon$, of polymer films of $\mathbf{P} \mathbf{3}$ and the annealed sample were evaluated by the measurement of refractive indices estimated from the ellipsometry method. Both samples showed relatively low $\varepsilon$.
\end{abstract}

Key words: Sliphenylenesiloxane, Cyclic structure, Glass transition temperature, Thermal degradation temperature, Dielectric constant.

\section{INTRODUCTION}

Polysilarylenesiloxane and their copolymers exhibit a high thermal degradation temperature, $T_{\mathrm{d}}$, and a high glass transition temperature, $T_{\mathrm{g}}$, compared with poly(dimethylsiloxane), PDMS, of a typical polysiloxane [1-5]. For example, poly(tetramethyl- $p$-silphenylenesiloxane), PTMPS, of a typical polysilarylenesiloxane showed relatively high $T_{\mathrm{d} 10}$ of $475^{\circ} \mathrm{C}$ and high $T_{\mathrm{g}}$ of $-20^{\circ} \mathrm{C}$ derived from phenylene groups in the backbone. In our previous work $[6,7]$, poly[(tetraphenyl- $p$-silphenylenesiloxane)-co-(tetramethyl- $p$-silphenylenesiloxane)],

PS-co-MS, was synthesized and the thermal properties were also investigated. PS-co-MS showed $458^{\circ} \mathrm{C}$ of $T_{\mathrm{d}}$ and $147^{\circ} \mathrm{C}$ of a softening point, $T_{\mathrm{s}}$. However, the thermal mechanical stability of these polymers for use as heat-resistant materials is not enough. Therefore, we have focused on the properties of PTMPS and conceived that the cyclization of silphenylenesiloxane moieties would improve further their thermal mechanical stability. Moreover, we expect that the relatively large intramolecular cavity is formed as a result of cyclization of silphenylenesiloxane moieties. If an intramolecular cavity is formed, such polymers would show several characteristics of low dielectric constant, low thermal conductivity, selective adsorption or transportation of gases and so on.

In this paper, the cyclic silphenylenesiloxane derivative having two allylhydrosilyl groups was prepared. Then, the hydrosilylation polymerization of this cyclic monomer in a dilute solution gave a unique polymer having alternate spiro-type structure of cyclic silphenylenesiloxane and cyclic carbosilane moieties. Thermal properties of the obtained polymers were investigated by using the thermogravimetric analysis,
TGA, and differential scanning calorimetry, DSC. Furthermore, the thin films of the polymers were prepared from a spin-coating method, and the dielectric constant was estimated by the ellipsometry method.

\section{EXPERIMENTAL}

\subsection{Materials}

Magnesium, $n$-hexane and methanol were purchased from Kanto Chemical. Triethylamine (Kanto Chemical) was distilled over $\mathrm{CaH}_{2}$ just before use. Tetrahydrofuran (THF), iodine, anhydrous sodium sulfate, toluene and acetonitrile were obtained from Wako Pure Chemical Industries. 1,4-Dibromobenzene, $0.10 \mathrm{~mol} \cdot \mathrm{L}^{-1}$ xylene solution of platinum 1,1,3,3-tetramethyl-1,3-divinyldisiloxane complex $(\mathrm{Pt}(\mathrm{dvs}))$ and palladium, $5 \mathrm{wt} . \%$ on activated carbon were purchased from Sigma-Aldrich. Dimethylchlorosilane was provided from Tokyo Chemical Industry. Allyldichlorosilane was obtained from Gelest Inc.

\subsection{Synthesis of 1,4-bis(dimethylsilyl)benzene (1)}

Magnesium (12.8 g, $528 \mathrm{mmol}$ ) was charged in a three-necked flask equipped with a dropping funnel and a condenser. The flask was dried in vacuo for $30 \mathrm{~min}$ heating with a heat gun. Then, argon was charged into the flask, and THF $(250 \mathrm{~mL})$ and iodine were added. After the mixture was vigorously stirred for $15 \mathrm{~min}$, dimethylchlorosilane $(58.0 \mathrm{~mL}, 522 \mathrm{mmol})$ was added. 1,4-Dibromobenzene (49.9 g, $211 \mathrm{mmol}$ ) was dissolved in THF $(200 \mathrm{~mL})$ and the solution was added dropwise to the flask at $0^{\circ} \mathrm{C}$. After stirring for $19 \mathrm{~h}$ at room temperature, the reaction mixture was washed with water $(300 \mathrm{~mL})$ and dried with anhydrous sodium sulfate. The solvent was removed via evaporation. Then, the product 
was distilled under reduced pressure to afford $\mathbf{1}$ as a colorless liquid. Yield: $64.9 \%$ b.p.: $600^{\circ} \mathrm{C} / 10 \mathrm{~Pa} .{ }^{1} \mathrm{H}$ NMR, $\delta\left(\mathrm{CDCl}_{3}, \quad\right.$ ppm, $\left.250 \mathrm{MHz}\right): 0.35(12 \mathrm{H}, \mathrm{d}$, $\left.\mathrm{Si}-\left(\mathrm{CH}_{3}\right)_{2}, J=3.8 \mathrm{~Hz}\right), 4.42$ (2H, sep, Si-H, $\left.J=3.8 \mathrm{~Hz}\right), 7.54$ (4H, s, Ph). IR, v ( $\mathrm{cm}^{-1}$, Reflect): 2958 (w, $\left.\mathrm{CH}_{3}\right), 2117$ (m, Si-H), $1250\left(\mathrm{~m}, \mathrm{Si}-\mathrm{CH}_{3}\right), 1136$ (m, Si-Ar), 866 (s), 831 (s).

2.3 Synthesis of 1,4-bis(hydroxydimethylsilyl)benzene (2)

Palladium, 5 wt. $\%$ on activated carbon $(0.334 \mathrm{~g}$, Pd: $0.157 \mathrm{mmol}$ ), was charged into a three-necked flask equipped with a dropping funnel. The flask was dried in vacuo and filled with argon. THF $(60.0 \mathrm{~mL})$ and water (4.64 mL, $258 \mathrm{mmol})$ were added to the flask. 1 (10.0 g, $51.4 \mathrm{mmol})$ was dissolved in THF $(70.0 \mathrm{~mL})$ and added dropwise to the flask. The reaction mixture was stirred for $16 \mathrm{~h}$ at room temperature. The catalyst was filtered off, and the solvent was removed via evaporation. The product was purified by recrystallization from the mixed solvents of THF and $n$-hexane, to obtain 2 as a white needle-like crystal. Yield: $91.7 \%{ }^{1} \mathrm{H}$ NMR, $\delta\left(\mathrm{CDCl}_{3}\right.$, ppm, 250MHz): $0.41\left(12 \mathrm{H}, \mathrm{s}, \mathrm{Si}-\left(\mathrm{CH}_{3}\right)_{2}\right), 1.83(2 \mathrm{H}, \mathrm{s}$, $\mathrm{Si}-\mathrm{OH}), 7.62$ (4H, s, Ph). IR, v ( $\mathrm{cm}^{-1}$, Reflect): 3190 (w, $\mathrm{Si}-\mathrm{OH}), 2956\left(\mathrm{w}, \mathrm{CH}_{3}\right), 1252\left(\mathrm{~m}, \mathrm{Si}-\mathrm{CH}_{3}\right), 1138(\mathrm{~m}$, Si-Ar), 866 (s), 818 (s).

2.4 Synthesis of the cyclic silphenylenesiloxane monomer (3)

$2(0.500 \mathrm{~g}, 2.21 \mathrm{mmol})$ was charged into a round-bottomed flask. The flask was dried in vacuo and filled with argon. THF $(220 \mathrm{~mL})$, triethylamine $(0.620$ $\mathrm{mL}, 4.42 \mathrm{mmol})$ and allyldichlorosilane $(0.290 \mathrm{~mL}, 2.21$ $\mathrm{mmol}$ ) were added to the flask. The reaction mixture was stirred for $24 \mathrm{~h}$ at room temperature. The triethylamine hydrochloride was filtered off, and the solvent was removed via evaporation. The product was purified with a silica gel column chromatography by using $n$-hexane as an eluent, to obtain $\mathbf{3}$ as a colorless liquid. Yield: $18.0 \%$. ${ }^{1} \mathrm{H}$ NMR, $\delta\left(\mathrm{CDCl}_{3}\right.$, ppm, 250MHz): $0.34(24 \mathrm{H}$, s, $\left.\mathrm{Si}-\left(\mathrm{CH}_{3}\right)_{2}\right), 1.64\left(4 \mathrm{H}, \mathrm{dd}, \mathrm{Si}-\mathrm{CH}_{2}-\mathrm{CH}, J=1.0 \mathrm{~Hz}\right.$, $6.8 \mathrm{~Hz}), 4.68\left(2 \mathrm{H}, \mathrm{d}, \mathrm{CH}=\mathrm{CH} \underline{\mathrm{H}}_{\text {trans }}, J=1.3 \mathrm{~Hz}\right), 4.94(2 \mathrm{H}, \mathrm{t}$, $\mathrm{Si}-\mathrm{H}, J=1.0 \mathrm{~Hz}), 5.00\left(2 \mathrm{H}, \mathrm{m}, \mathrm{CH}=\mathrm{CH}_{\mathrm{cis}}\right), 5.79(2 \mathrm{H}, \mathrm{m}$, $\left.\mathrm{CH}=\mathrm{CH}_{2}\right), 7.34(8 \mathrm{H}, \mathrm{s}, \mathrm{Ph}) . \mathrm{IR}, v\left(\mathrm{~cm}^{-1}\right.$, Reflect): 2958 (w, $\mathrm{CH}_{3}$ ), 2146 (w, Si-H), 1631 (w, $\mathrm{CH}=\mathrm{CH}_{2}$ ), 1254 (m, Si-CH ${ }_{3}$, 1140 (m, Si-Ar), 1053 (s, Si-O-Si), 810 (s), 773 (s).

\subsection{Hydrosilylation polymerization of $\mathbf{3}$}

A typical polymerization procedure was described below. 3 (0.0933 g, $0.158 \mathrm{mmol})$ was charged into a round-bottomed flask. The flask was dried in vacuo and filled with argon. Toluene $(15.8 \mathrm{~mL})$ and $\mathrm{Pt}(\mathrm{dvs})(1.58$ $\mu \mathrm{L})$ were added to the flask. The reaction mixture was stirred at $80^{\circ} \mathrm{C}$ for $72 \mathrm{~h}$. The solvent was removed via evaporation. The polymer was dissolved in THF of 0.5 $\mathrm{mL}$ and precipitated into methanol cooled by acetone/dry ice. P3 was obtained as a white powder. Yield: $41.8 \%$. ${ }^{1} \mathrm{H} \mathrm{NMR}, \delta\left(\mathrm{CDCl}_{3}, \mathrm{ppm}, 500 \mathrm{MHz}\right): 0.34(24 \mathrm{H}, \mathrm{s}$, $\left.\mathrm{Si}-\left(\mathrm{CH}_{3}\right)_{2}\right), 0.69\left(8 \mathrm{H}, \mathrm{s}, \mathrm{Si}-\mathrm{CH}_{2}\right), 1.56\left(4 \mathrm{H}, \mathrm{s}, \mathrm{CH}_{2}\right), 7.33$ (8H, s, Ph). IR, v ( $\mathrm{cm}^{-1}$, Reflect): 2956 (w, $\left.\mathrm{CH}_{3}\right), 1252$ (m, Si-CH ${ }_{3}$ ), 1138 (m, Si-Ar), 1039 (s, Si-O-Si), 818 (s), 773 (s).

\subsection{Characterizations}

${ }^{1} \mathrm{H}$ NMR spectra were recorded on a Bruker DPX-250 or DRX-500 NMR spectrometer. IR spectra were recorded with Horiba FT-720 equipped with SensIR technologies DuraSamplIR II. High performance liquid chromatography (HPLC) analysis was conducted with a Waters 2690 by using $20 \mathrm{v} / \mathrm{v} \% \mathrm{THF} / \mathrm{CH}_{3} \mathrm{CN}$ as an eluent, equipped with a column of ODS-3. GPC analysis was conducted with a Tosoh GPC-8020 by using THF as an eluent, equipped with four columns of Tskgel SuperH5000, 3000, 2000 and 1000. Standard polystyrenes were used for calibration.

\subsection{Thermal analysis}

TGA was performed by using a SII Nano Technology TG/DTA6200 at a heating rate of $10^{\circ} \mathrm{C} / \mathrm{min}$ under nitrogen $(100 \mathrm{~mL} / \mathrm{min})$ or air flow $(50 \mathrm{~mL} / \mathrm{min})$. DSC was performed by using a SII Nano Technology DSC6220 at a heating rate of $10^{\circ} \mathrm{C} / \mathrm{min}$ under nitrogen flow $(30 \mathrm{~mL} / \mathrm{min})$.

2.8 Measurements of the thicknesses and the refractive indices

The polymers were spin-coated from 1 wt.\% solution in toluene on silicon wafer with a spin coater Active ACT-300A for $30 \mathrm{sec}$ at $500 \mathrm{rpm}$. Refractive indices and thicknesses of polymer films were measured by using the standard ellipsometric method on a Mizojiri DHA-XAS at wavelength of $632.8 \mathrm{~nm}$, and at an incident irradiation angle of $70^{\circ}$.

\section{RESULTS AND DISCUSSION}

3.1 Synthesis of monomers

The synthetic route of cyclic silphenylenesiloxane monomer having two allylhydrosilyl groups is shown in Scheme 1 with polymerization reaction. The starting compound, 1, was synthesized via Grignard reaction of $p$-dibromobenzene with dimethylchlorosilane, where iodine was used to activate magnesium. Then, $\mathbf{2}$ was synthesized nearly quantitively by the hydrolysis of $\mathbf{1}$, and 3 was synthesized in a dilute solution by the cyclization of $\mathbf{2}$ with allyldichlorosilane using triethylamine as a base. Because of the difficulty in ionizing of $\mathbf{3}$, the characterization of products by using gas chromatography and mass spectrometry was unsuccessful. Therefore, HPLC analysis was employed to characterize the products. HPLC data suggested that the cyclic oligomers of larger ring size were generated as biproducts to obtain 3 in the low yield $(18.0 \%)$. Moreover, 3 has two kinds of diastereomers, however, either cis or trans compound was obtained nearly selectively $(\sim 90 \%)$.

\subsection{Hydrosilylation polymerization}

The hydrosilylation polymerizations of the monomer compound, 3, were carried out in the different concentrations to prevent a cross-linking reaction, which were summarized in Table I. The conditions and the results of hydrosilylation indicated that a cross-linking reaction would partially occur. P1 was synthesized in 1.0 $\mathbf{M}$ solution of the monomer. In this case, P1 was obtained as a completely insoluble yellowish-brown solid, which was confirmed by washing the polymer with THF to give no soluble part. On the other hand, the 
Table I. Conditions and results of polymerization.

\begin{tabular}{|c|c|c|c|c|c|c|c|}
\hline \multirow{2}{*}{ Polymer } & \multirow{2}{*}[3]{$_{0}\left(\mathrm{~mol}^{-\mathrm{L}^{-1}}\right)$} & \multirow{2}{*}{ ) Temp. $\left({ }^{\circ} \mathrm{C}\right)$} & \multirow{2}{*}{ Time $(\mathrm{h})$} & \multicolumn{2}{|c|}{ Yield (\%) } & \multirow{2}{*}{$\left.M_{\mathrm{n}} \cdot 10^{-4} \mathrm{~b}\right)$} & \multirow{2}{*}{$M_{\mathrm{w}} \cdot M_{\mathrm{n}}^{-1 \mathrm{~b})}$} \\
\hline & & & & Soluble part & Insoluble part & & \\
\hline P1 & 1.00 & 100 & 19 & - & 69.8 & - & - \\
\hline P2 & 0.10 & 100 & 24 & $8.92^{c)}$ & 46.1 & 3.34 & 4.79 \\
\hline P3 & 0.01 & 80 & 72 & 41.8 & - & 1.04 & 2.09 \\
\hline
\end{tabular}

a) $[3]_{0}$ means the initial concentration of the monomer 3 .

b) $M_{\mathrm{n}}$ (number-average molecular weight) and $M_{\mathrm{w}}$ (weight-average molecular weight) were determined by GPC.

c) A soluble part was obtained by the Soxhlet extraction method from the insoluble product.

soluble part was obtained by a Soxhlet extraction method using THF as an eluent from P2. GPC analysis of a soluble part of $\mathbf{P} 2$ showed the high polydispersity of $M_{\mathrm{w}} \cdot M_{\mathrm{n}}^{-1}$. It was suggested from this result that the cross-linking reaction would occur even in $0.1 \mathrm{M}$ dilute solution and the part having relatively low degree of cross-linking was dissolved in THF. Therefore, $\mathbf{P 3}$ was synthesized in the more dilute solution $(0.01 \mathrm{M})$. As a result, P3 was obtained as a soluble powder and showed relatively low polydispersity, as shown in Table I. Consequently, when this reaction proceeds in such a enough dilute solution, the cross-linking reaction would not occur. The IR spectra of monomer and polymers are shown in Fig. 1. The evidence of successful hydrosilylation was confirmed by the disappearance of the peaks at 2146,1631 and $880 \mathrm{~cm}^{-1}$, corresponding to $\mathrm{Si}-\mathrm{H}$ stretching, $\mathrm{C}=\mathrm{C}$ of allyl groups and $\mathrm{Si}-\mathrm{H}$ deformation, respectively. Moreover, the other peaks were unchanged, which indicates that these polymers maintained the cyclic structure arising from the monomer unit throughout the polymerization. The ${ }^{1} \mathrm{H}$ NMR spectrum of $\mathbf{P 3}$ is also shown in Fig. 2. The signals arising from $\mathrm{CH}_{3}$ (a), $\mathrm{CH}_{2}$ (b) adjacent to $\mathrm{Si}, \mathrm{CH}_{2}$ (c) and phenyl proton (d) can be seen in Fig. 2. However, a few allyl and hydrosilyl groups remained after polymerization, where the signals derived from them were observed at 4.8 6.3 ppm in Fig. 2.

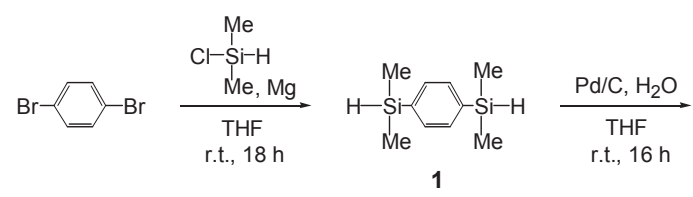

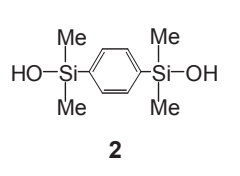
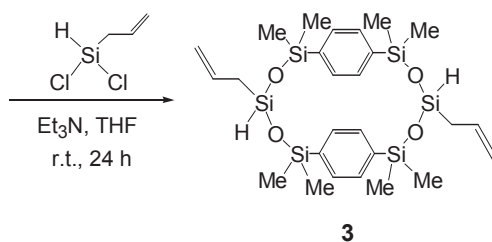

3

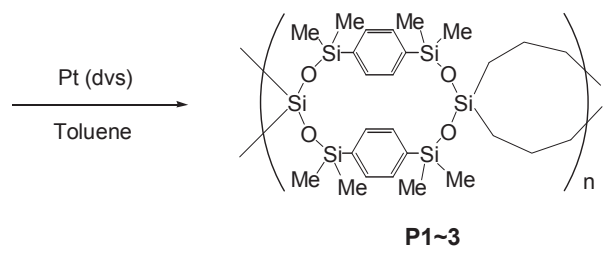

Scheme 1. Syntheses of monomer and polymers.
On the other hand, when $\mathbf{P 3}$ was heated to $200^{\circ} \mathrm{C}$ at a heating rate of $10^{\circ} \mathrm{C} / \mathrm{min}$ under argon flow, $\mathbf{P 3}$ became insoluble to common solvents. The IR spectrum of the heated sample was quite similar to that of $\mathbf{P 3}$, and thus P3 maintained the cyclic structure throughout the heating to $200^{\circ} \mathrm{C}$. These results suggested that the cross-linking reaction such as an intermolecular hydrosilylation reaction between the remained allyl and hydrosilyl groups in $\mathbf{P 3}$ occurred by heating to afford an insoluble polymer, which was also the evidence that the allyl and hydrosilyl groups of $\mathbf{3}$ remained and the linear alkylene chain existed except cyclic carbosilane moiety in the polymerization of $\mathbf{3}$.

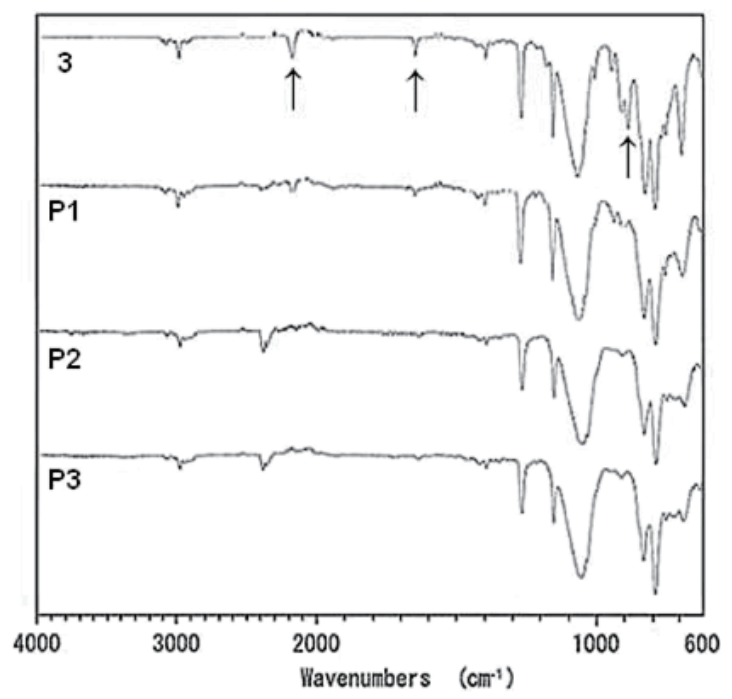

Fig. 1. IR spectra of monomer and polymers.

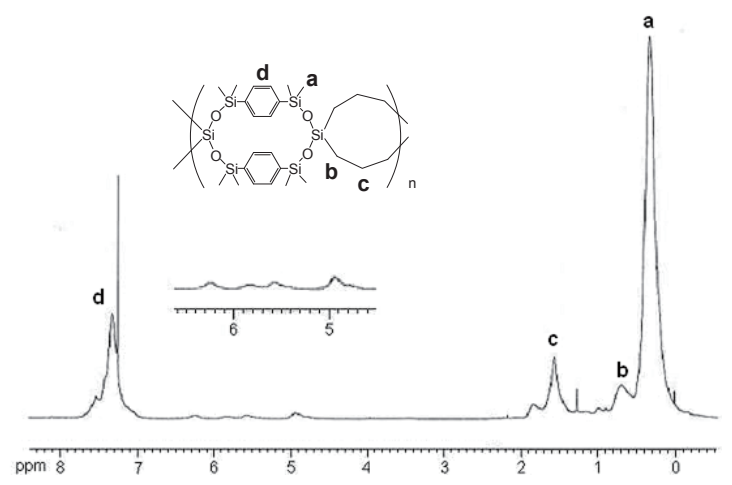

Fig. 2. ${ }^{1} \mathrm{H}$ NMR spectrum of $\mathbf{P 3}$. 
3.3 Thermal properties of polymers

The glass transition temperature $\left(T_{\mathrm{g}}\right)$ and the degradation temperature $\left(T_{\mathrm{d}}\right)$ of polymers are listed in Table II. The $T_{\mathrm{g}}$ of $\mathbf{P 1}$ was observed at around room temperature, whereas $\mathbf{P 3}$ showed relatively high $T_{\mathrm{g}}$. Concerning the larger amount of cyclic carbosilane moiety in soluble $\mathbf{P 3}$ than highly cross-linked insoluble $\mathbf{P 1}$, it is suggested that the cyclic structure makes a polymer chain rigid and consequently improves a thermal and mechanical stability. In addition, the $T_{\mathrm{g}}$ of P3 sample heated to $200^{\circ} \mathrm{C}$ under nitrogen flow was not observed below the degradation temperature. Therefore, P3 might be suitable for a heat-resistant material such as high temperature coating.

The TG curves of $\mathbf{P 1}$ and $\mathbf{P 3}$ are shown in Fig. 3. In nitrogen atmosphere, both polymers showed a high residue yield at $1000^{\circ} \mathrm{C}, W_{\mathrm{r} 1000}$, due to the cyclic structure, and the main thermal degradation of these polymers started above $440^{\circ} \mathrm{C}$. The main thermal degradation of these polymers in air started above $380^{\circ} \mathrm{C}$, which are considerably lower than the $T_{\mathrm{d} 5}$ in nitrogen. The $W_{\text {r1000 }}$ values in air were also lower than those in nitrogen. Furthermore, in the case of $\mathbf{P 3}$, no weight loss was observed at around $200^{\circ} \mathrm{C}$. Therefore, it was considered that the cross-linking reaction, which was caused by the remained allyl and hydrosilyl groups, mainly occurred at around $200^{\circ} \mathrm{C}$ to afford insoluble $\mathbf{P 3}$.

3.4 Dielectric constants of polymer films

The refractive indices of polymer films are listed in Table III. The calculated dielectric constant, $\varepsilon$, according

Table II. Thermal properties of polymers.

\begin{tabular}{|c|c|c|c|c|}
\hline Polymer & {$[\mathrm{M}]_{0}\left(\mathrm{~mol}^{-\mathrm{L}^{-1}}\right)$} & $T_{\mathrm{g}}\left({ }^{\circ} \mathrm{C}\right)^{\mathrm{a})}$ & $T_{\mathrm{d} 5}\left({ }^{\circ} \mathrm{C}\right)^{\mathrm{b})}$ & $W_{\mathrm{r} 1000}(\%)^{\mathrm{b})}$ \\
\hline P1 & 1.00 & 24.0 & 484 & 78.2 \\
\hline P3 & 0.01 & 76.5 & 446 & 73.4 \\
\hline PTMPS & $\longrightarrow$ & -20.0 & 485 & 27.4 \\
\hline \multicolumn{5}{|c|}{$\begin{array}{l}\text { a) } T_{g} \text { (glass transition temperature) was determined by DSC on the second } \\
\text { heating process under } \mathrm{N}_{2} \text {. }\end{array}$} \\
\hline \multicolumn{5}{|c|}{$\begin{array}{l}\text { b) } T_{\mathrm{d} 5}\left(5 \% \text { weight loss temperature) and } W_{\mathrm{r} 1000} \text { (residue yield at } 1000^{\circ} \mathrm{C}\right) \\
\text { were determined by TG curve under } \mathrm{N}_{2} \text {. }\end{array}$} \\
\hline
\end{tabular}

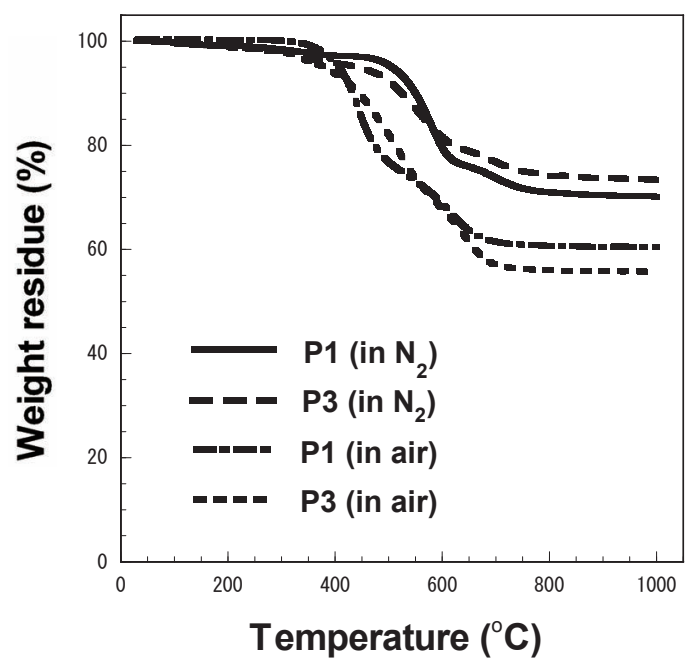

Fig. 3. TG curves of polymers.
Table III. The refractive indices and the dielectric constants of polymer films.

\begin{tabular}{ccc}
\hline Polymer & $n^{\text {a) }}$ & $\varepsilon^{\text {b) }}$ \\
\hline P3 & 1.56 & 2.42 \\
P3' $^{\text {c) }}$ & 1.57 & 2.45 \\
\hline
\end{tabular}

\footnotetext{
a) Determined by ellipsometric method at wavelength of $632.8 \mathrm{~nm}$.

b) $\varepsilon$ (dielectric constant) was estimated by the refractive index measurement.

c) P3' was obtained by annealing P3 at $200{ }^{\circ} \mathrm{C}$ for $1 \mathrm{~h}$ under air.
}

to the Maxwell's equation, $\varepsilon=n^{2}$, are also listed in Table III. P3 showed the lower $\varepsilon$ value than conventional $\mathrm{SiO}_{2}$ of $\varepsilon=3.9$ and polyimides of $\varepsilon=3.1-3.5$ [8]. This result indicated that the introduction of cyclic silphenylenesiloxane moieties into polymer backbone produced the relatively large intramolecular cavity. Fortunately, $\mathbf{P 3}$ annealed at $200^{\circ} \mathrm{C}$ for $1 \mathrm{~h}$ under air (P3') also showed relatively low $\varepsilon$. Therefore, $\mathbf{P} 3$ might be suitable to use as heat-resistant materials exhibiting a low $\varepsilon$.

\section{CONCLUSIONS}

A novel cyclic silphenylenesiloxane derivative having two allylhydrosilyl groups was synthesized. The spiro-type polymer $\mathbf{P 3}$ having the alternate structure of cyclic silphenylenesiloxane and cyclic carbosilane moieties by the hydrosilylation polymerization of the cyclic monomer in a dilute solution. The thermal properties of the obtained spiro-type polymer were investigated by using TGA and DSC. As a result, P3 showed relatively high $T_{\mathrm{g}}$ and the large amount of weight residue at $1000^{\circ} \mathrm{C}$ derived from the cyclic structure. In addition, the annealed $\mathbf{P 3}$ at $200^{\circ} \mathrm{C}$ became insoluble to common solvents and showed the high thermal and mechanical stability, which also showed a low $\varepsilon$. Consequently, $\mathbf{P} 3$ might be suitable to be used as heat-resistant materials for high temperature coating and low $\varepsilon$ materials.

\section{REFERENCES}

[1] M. Ikeda, T. Nakamura, Y. Nagase, K. Ikeda and Y. Sekine, J. Polym. Sci., Polym. Chem. Ed., 19, 2595 (1981).

[2] C. U. Pittman, W. J. Patterson and S. P. McManus, J. Polym. Sci., Polym. Chem. Ed., 14, 1715 (1976).

[3] J. M. Funt, R. D. Parekh, J. H. Magill and Y. T. Shah, J. Polym. Sci., Polym. Chem. Ed., 13, 2181 (1975).

[4] R. L. Merker, M. J. Scott and G. G. Haberland, J. Polym. Sci., Polym. Chem. Ed., 2, 31 (1964).

[5] N. Okui, H. M. Li and J. H. Magill, Polymer, 19, 411 (1978).

[6] H. Ito, E. Akiyama, Y. Nagase and S. Fukui, Polym. J., 39, 471 (2007).

[7] H. Ito, E. Akiyama, Y. Nagase and S. Fukui, Trans. Mater. Res. Soc. Jpn., 31, 1065 (2006).

[8] G. D. Fu, B. Y. Zong, E. T. Kang, K. G. Neoh, C. C. Lin and D. J. Liaw, Ind. Eng. Chem. Res., 43, 6723 (2004).

(Received December 9, 2008; Accepted April 17, 2009) 